\title{
MODELLING OF ELECTROMECHANICAL COUPLING IN GEOMETRICALLY EXACT BEAM DYNAMICS
}

\author{
Dengpeng Huang, Sigrid Leyendecker \\ Institute of Applied Dynamics, Friedrich-Alexander-Universität Erlangen-Nürnberg \\ Immerwahrstrasse 1, 91058 Erlangen, Germany \\ dengpeng.huang@fau.de, sigrid.leyendecker@fau.de
}

Key words: Dielectric Elastomer Actuator, Variational Integrator, Electromechanical Coupling, Geometrically Exact Beam

\begin{abstract}
Dielectric elastomers show promising performance as actuators for soft robotics. Thus, accurate and efficient numerical algorithms for the simulation of Dielectric Elastomer Actuators (DEAs) are required for the design and control of the soft robotic system. In this work, the Cosserat formulation of geometrically exact beam dynamics is extended by adding the electric potential as an additional degree of freedom to account for the electrical effects. A formulation of electric potential and electric field for the geometrically exact beam model is proposed such that complex beam deformations can be generated by the electrical forces. The kinematic variables in continuum electromechanics are formulated in terms of beam strains. The electromechanically coupled constitutive model for the beam formulation is obtained by integrating the strain energy in continuum electromechanics over the beam cross section, which leads to a direct transfer of the dielectric constitutive models in continuum mechanics to the beam model. The electromechanically coupled beam dynamics is solved with a variational time integrator scheme. By applying different electrical boundary conditions to the beam nodes, different deformation modes of the beam are obtained in the numerical example.
\end{abstract}

\section{INTRODUCTION}

Due to their better performances in energy efficiency, completing complex movements and safe interaction with the environment, soft robotic systems are highly demanded in industrial production, medical treatment and daily life. As a promising actuation approach for soft robotics, the Dielectric Elastomer Actuators (DEAs) have been developed to serve as artificial muscles, see e.g. $[4,12,10]$ and [8]. In the DEA cell, the dielectric elastomer is sandwiched between two compliant electrodes. By applying the external electric potential to the electrodes, the electric field is induced within the DEA cell and the dielectric material is polarized, resulting in an electrostatic pressure, see the models in $[14,22,20]$ and [16] for instance. The contractive pressure leads to the contraction of the DEA such that it can be applied as an actuator. The DEAs are essentially composed by multiple stacked capacitors.

The deformation behavior of the DEA is governed by the electromechanical coupling in the dielectric material. In the past years, much effort has been made to address the electromechanical coupling problem, see e.g. the theory of interaction of electromagnetic and elastic fields in deformable continua in [13], the nonlinear electroelasticity formulation for the finite deformation in [7] and the variational formulations of the electro- and magneto-elastostatics in [21]. Additionally, the constitutive models for the 
dielectric elastomers have been investigated, see e.g. [23, 21] and [19]. In [17], a viscoelastic 3D finite element model of the DEA is developed for the dynamic analysis within a structure preserving time integration scheme. This model is extended to flexible multibody system dynamics in [15].

In large 3D finite element models, huge amounts of degrees of freedom are usually required, which leads to inefficient simulation of DEA. To simulate the long thin artificial muscles like the stacked DEA, 3D finite element model can be replaced by the reduced models, such as the geometrically exact beam. The geometrically exact beam performs well concerning the tradeoff between computational cost and accuracy. The Cosserat beam [6] is formulated by assigning the rotational degrees of freedom to points in continuum, which leads to a consistent formulation of flexible multibody systems. The fundamental formulations on geometrically exact beam can be found in [18] and [2] for instance.

In this work, an electromechanically coupled beam model for the simulation of stacked dielectric elastomer actuators is developed, where the Cosserat beam formulation is obtained by relating the beam kinematics to that in continuum electromechanics. The explicit beam specific expressions for this coupled problem are discussed in [9]. A formulation of electric potential and electric field for the geometrically exact beam model is proposed such that complex beam deformations can be generated by the electrical forces. The deformation gradient and electric field are formulated in terms of beam strains. The electromechanically coupled constitutive model for the beam formulation is obtained by integrating the strain energy in continuum electromechanics over the beam cross section, which leads to the direct application of the material models in continuum electromechanics to the beam model. The viscoelastic effect is taken into account in the non-conservative force term. The electromechanically coupled problem

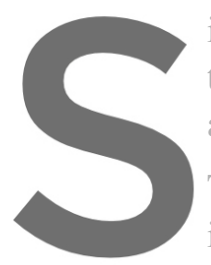
in beam dynamics is fir time integration. By apply are obtained in the numerica

This paper is structured as in continuum electromech
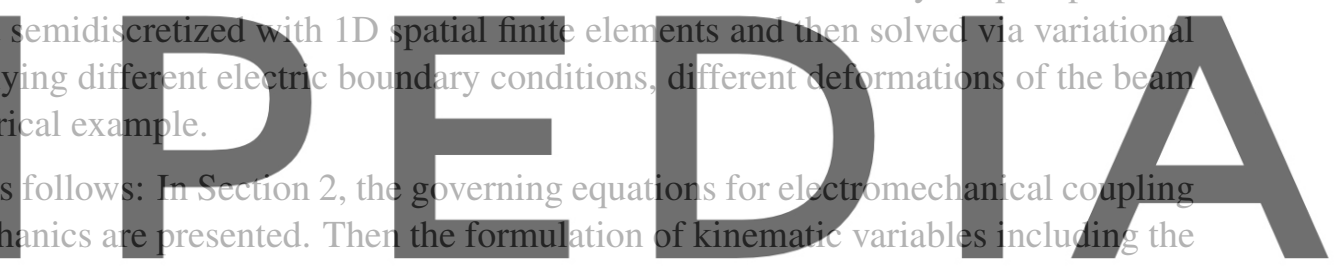

deformation gradient, the electric potential and the electric field are derived for the beam in Section 3.

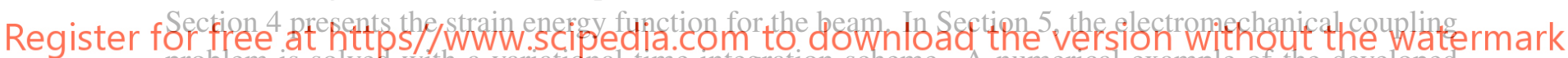
problem is solved with a variational time integration scheme. A numerical example of the developed model is presented in Section 6, followed by the conclusions in Section 7.

\section{GOVERNING EQUATIONS FOR ELECTROMECHANICAL COUPLING}

We consider a dielectric elastic solid occupying the domain $B \subset \mathbb{R}^{3}$, where the boundary $\partial B$ of the solid is composed by the Dirichlet type sections $\partial_{u} B$ and $\partial_{\phi} B$, and the Neumann type sections $\partial_{\sigma} B$ and $\partial_{D} B$. The position of a material point in the deformed configuration at time $t$ is given by the motion

$$
\mathbf{x}=\mathbf{X}+\mathbf{u}(\mathbf{X}, t)
$$

where $\mathbf{X}$ is the position of the material point in the initial configuration and $\mathbf{u}$ is the displacement. The deformation of the solid is governed by the balance law of momentum and the Maxwell equations. In this part, the governing equations of continuum electromechanics are summarized. The corresponding beam equations are formulated in [9]. 


\subsection{Balance of linear and angular momentum}

The balance law of linear momentum and the mechanical boundary conditions in the electro-elastodynamic process is given by

$$
\begin{aligned}
\nabla_{\mathbf{X}} \cdot \mathbf{P}+\rho_{0} \overline{\mathbf{b}} & =\rho_{0} \ddot{\mathbf{u}} & & \text { in } \quad B, \\
\mathbf{u} & =\overline{\mathbf{u}} & & \text { on } \quad \partial_{u} B, \\
\mathbf{P} \cdot \mathbf{N} & =\overline{\mathbf{T}} & & \text { on } \quad \partial_{\sigma} B,
\end{aligned}
$$

with $\mathbf{P}$ the first Piola-Kirchhoff stress tensor, $\rho_{0}$ the mass density in initial configuration, $\overline{\mathbf{b}}$ the body force vector, $\ddot{\mathbf{u}}$ the acceleration, $\overline{\mathbf{u}}$ the prescribed displacement, $\mathbf{N}$ the outward unit normal vector and $\overline{\mathbf{T}}$ the prescribed traction. The local balance of angular momentum reads

$$
\mathbf{F P}^{T}=\mathbf{P F}^{T},
$$

in which $\mathbb{F}$ is the deformation gradient defined as $\mathbb{F}=\partial \mathbf{x}(\mathbf{X}, t) / \partial \mathbf{X}$.

\subsection{Maxwell equations}

By neglecting the magnetic field, the Maxwell equations and the electrical boundary conditions are given by

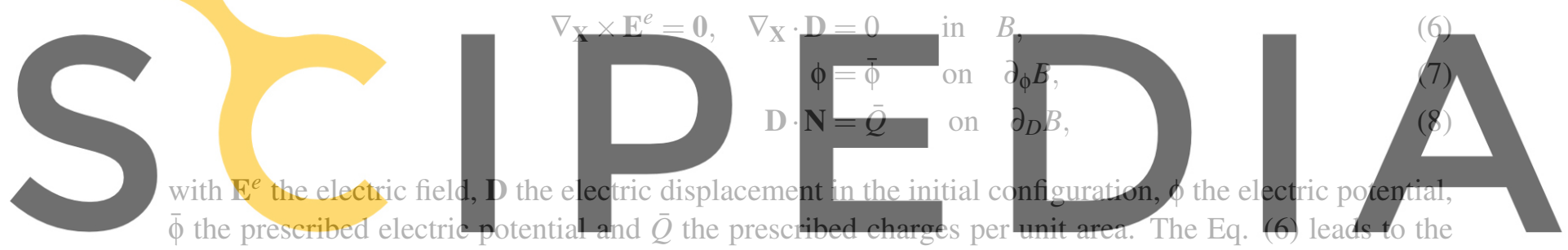

definition of the electric field as the gradient of a scalar electric potential

Register for free at https//www.scipedia.com to download the version without the watermark

$$
\mathbf{E}^{e}=-\frac{\partial \phi}{\partial \mathbf{X}}
$$

\subsection{Electromechanical coupling}

By imposing the external electric field on the body of dielectric elastomer, the contractive pressure is induced and thus the deformation of the body is generated. The electromechanically coupled constitutive behavior of the DEA is described by the strain energy function $\Omega\left(\mathbf{F}, \mathbf{E}^{e}\right)$ with the additive form

$$
\Omega\left(\mathbf{F}, \mathbf{E}^{e}\right)=\Omega^{m}(\mathbf{F})+\Omega^{\mathrm{em}}\left(\mathbf{F}, \mathbf{E}^{e}\right)+\Omega^{e}\left(\mathbf{E}^{e}\right)
$$

with $\Omega^{m}(\mathbf{F})$ referring to the purely mechanical behavior, $\Omega^{\mathrm{em}}\left(\mathbf{F}, \mathbf{E}^{e}\right)$ referring to the electomechanical coupling and $\Omega^{e}\left(\mathbf{E}^{e}\right)$ referring to the pure electric behavior. Accordingly, the constitutive equations are given by

$$
\mathbf{D}=-\rho_{0} \frac{\partial \Omega\left(\mathbf{F}, \mathbf{E}^{e}\right)}{\partial \mathbf{E}^{e}}, \quad \mathbf{P}=\rho_{0} \frac{\partial \Omega\left(\mathbf{F}, \mathbf{E}^{e}\right)}{\partial \mathbf{F}}
$$




\section{RELATION OF THE BEAM AND CONTINUUM KINEMATICS}

To apply the material model $\Omega\left(\mathbf{F}, \mathbf{E}^{e}\right)$ of DEA to the beam, the kinematic variables $\mathbf{F}$ and $\mathbf{E}^{e}$ have to be formulated in terms of the beam strain measures.

\subsection{Mechanical kinematics of the beam}

As shown in Fig. 1, the deformation of an initially straight beam is distinguished by the initial configuration and the current configuration, where the origin $\mathbf{O}$ of the global Cartesian coordinate system is set to one end of the beam. The body of the beam is described by a family of cross sections. The cross-section centroids are connected by a curve also known as line of centroids. The arc-length of the line of centroids in the initial configuration is denoted by $s \in I \subset \mathbb{R}$. At each point of the curve $s \in I \rightarrow \varphi(s, 0) \in \mathbb{R}^{3}$, the orientation of the cross section is defined by an orthogonal frame $\mathbf{d}_{i}(s, 0),(i=1,2,3)$.
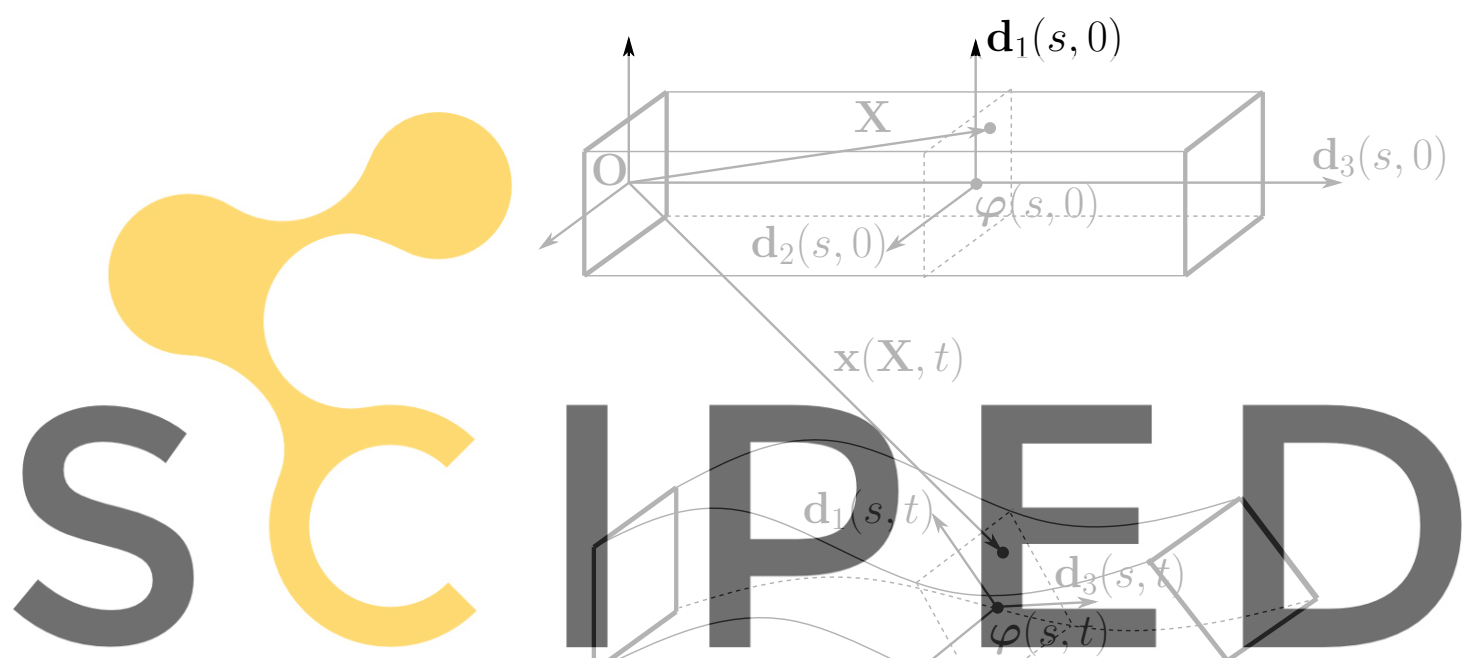

\section{Register for free at https//www.scipedia.com to download the version without the watermark}

Figure 1: Initially straight and deformed configuration of the beam.

The position of a material point on the cross section at $s$ can be described in the initial configuration as

$$
\mathbf{X}\left(X^{k}, s\right)=\varphi(s, 0)+X^{k} \mathbf{d}_{k}(s, 0), \quad k=1,2 .
$$

The deformation gradient at a point $\left(X^{1}, X^{2}, s\right)$ in the beam can be written as, see [3],

$$
\begin{aligned}
\mathbf{F}\left(X^{1}, X^{2}, s, t\right) & =\frac{\partial \mathbf{x}}{\partial \mathbf{X}}=\frac{\partial \mathbf{x}}{\partial X_{i}} \otimes \mathbf{d}_{i}(s, 0) \\
& =\left[\mathbf{I}+\left(\frac{\partial \varphi(s, t)}{\partial s}-\mathbf{d}_{3}(s, t)+X^{1} \frac{\partial \mathbf{d}_{1}(s, t)}{\partial s}+X^{2} \frac{\partial \mathbf{d}_{2}(s, t)}{\partial s}\right) \otimes \mathbf{d}_{3}(s, t)\right] \Lambda(s)
\end{aligned}
$$

with the rotation tensor $\Lambda(s)=\mathbf{d}_{i}(s, t) \otimes \mathbf{d}_{i}(s, 0)$ and $\Lambda(s)^{-1}=\Lambda(s)^{T}$. Since the derivatives in Eq. (13) are related to the beam strains $\kappa$ and $\gamma$ by

$$
\frac{\partial \mathbf{d}_{k}(s, t)}{\partial s}=\kappa(s, t) \times \mathbf{d}_{k}(s, t)=\left(\kappa_{j} \mathbf{d}_{j}\right) \times \mathbf{d}_{k},(k=1,2) \quad \frac{\partial \varphi(s, t)}{\partial s}-\mathbf{d}_{3}(s, t)=\gamma(s, t),
$$


the deformation gradient $\mathbf{F}$ in Eq. (13) can be further formulated in terms of the beam strain measures as

$$
\mathbf{F}=\left[\mathbf{I}+\left(\gamma(s, t)+\kappa(s, t) \times X^{k} \mathbf{d}_{k}(s, t)\right) \otimes \mathbf{d}_{3}(s, t)\right] \Lambda(s)=\left[\mathbf{I}+\mathbf{a}(s, t) \otimes \mathbf{d}_{3}(s, t)\right] \Lambda(s)
$$

with $\mathbf{a}=\gamma+\kappa \times X^{k} \mathbf{d}_{k}$ in the current configuration. The determinant of the deformation gradient reads

$$
J=\operatorname{det}(\mathbf{F})=1+\mathbf{a}(s, t) \cdot \mathbf{d}_{3}(s, t) .
$$

The material time derivative of the location of a material point in the current configuration reads

$$
\dot{\mathbf{x}}\left(X^{k}, s, t\right)=\dot{\varphi}(s, t)+X^{k} \dot{\mathbf{d}}_{k}(s, t),
$$

by which the time derivative of deformation gradient can be evaluated with

$$
\begin{aligned}
\dot{\mathbf{F}}\left(X^{1}, X^{2}, s, t\right) & =\frac{\partial \dot{\mathbf{x}}}{\partial \mathbf{X}}=\frac{\partial \dot{\mathbf{x}}}{\partial X_{i}} \otimes \mathbf{d}_{i}(s, 0) \\
& =\dot{\Lambda}(s)+\left(\frac{\partial \dot{\varphi}(s, t)}{\partial s}-\dot{d}_{3}(s, t)+X^{1} \frac{\partial \dot{d}_{1}(s, t)}{\partial s}+X^{2} \frac{\partial \dot{d}_{2}(s, t)}{\partial s}\right) \otimes \mathbf{d}_{3}(s, 0) .
\end{aligned}
$$

\subsection{Electrical kinematics in the beam}

In this work, the electric potential at the point $\left(X^{1}, X^{2}, s\right)$ is formulated by the electric potential at the

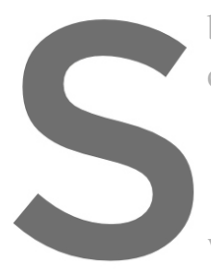

beam node plus the incr

cross section as shown

with $\phi_{0}(s, 0)$ the electrid p
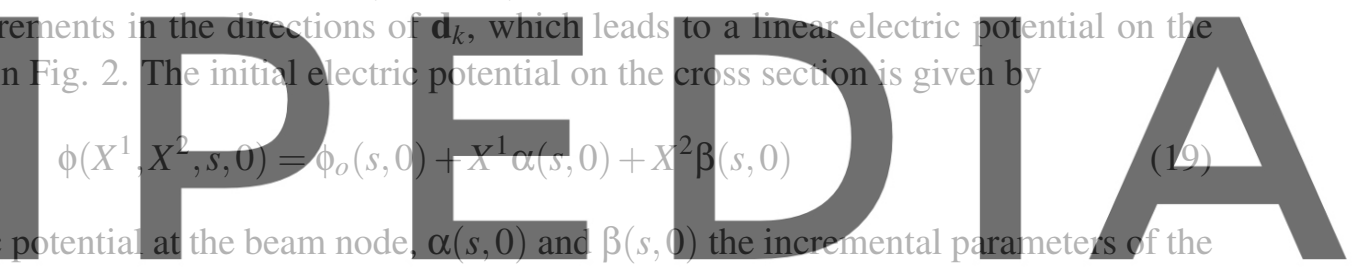

electric potential in the directions of $\mathbf{d}_{1}(s, 0)$ and $\mathbf{d}_{2}(s, 0)$, respectively. According to Eq. (9), the electric

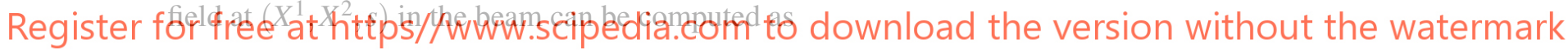

$$
\begin{aligned}
\mathbb{E}^{e} & =-\frac{\partial \phi(s, t)}{\partial X_{i}} \otimes \mathrm{d}_{i}(s, 0) \\
& =-\left[\alpha(s, t) \mathbf{d}_{1}(s, 0)+\beta(s, t) \mathbf{d}_{2}(s, 0)+\left(\frac{\partial \phi_{o}(s, t)}{\partial s}+X^{1} \frac{\partial \alpha(s, t)}{\partial s}+X^{2} \frac{\partial \beta(s, t)}{\partial s}\right) \mathbf{d}_{3}(s, 0)\right] .
\end{aligned}
$$

\section{STRAIN ENERGY FUNCTION FOR THE BEAM}

To apply the material models of continuum electromechanics to a beam formulation, the rewriting of the strain energy function in terms of the beam strains is required, which can be achieved by applying the kinematic relations introduced in Section 3. The extended Neo-Hookean model for the dielectric elasticity in [17] is adopted in this work, where the strain energy density is given by

$$
\Omega\left(\mathbf{C}, \mathbf{E}^{e}\right)=\underbrace{\frac{\mu}{2}(\mathbf{C}: \mathbf{1}-3)-\mu \ln J+\frac{\lambda}{2}(\ln J)^{2}}_{\text {Neo-Hookean }}+\underbrace{c_{1} \mathbf{E}^{e} \cdot \mathbf{E}^{e}+c_{2} \mathbf{C}:\left(\mathbf{E}^{e} \otimes \mathbf{E}^{e}\right)}_{\text {Polarization in dielectric material }}-\underbrace{\frac{1}{2} \varepsilon_{0} J \mathbf{C}^{-1}:\left(\mathbf{E}^{e} \otimes \mathbf{E}^{e}\right)}_{\text {Free space term in vacuum }}
$$




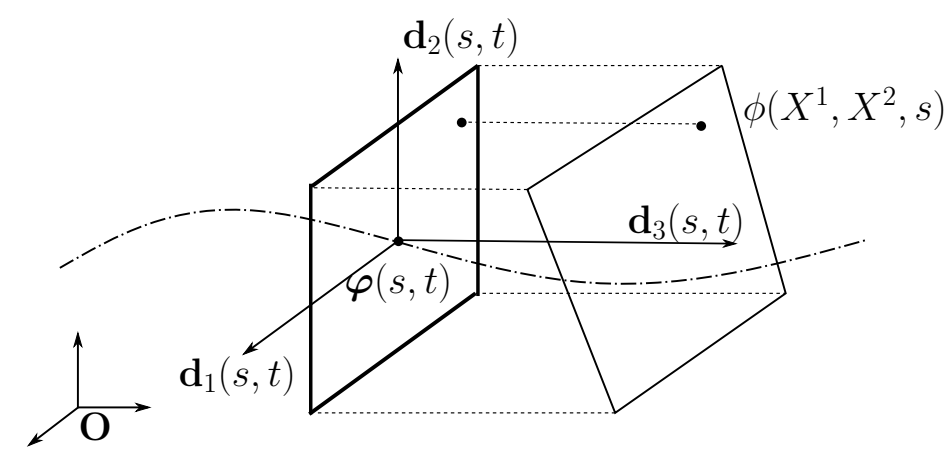

Figure 2: Linear distribution of electric potential on a cross section.

with $\varepsilon_{0}$ the vacuum permittivity, $c_{1}$ and $c_{2}$ the electrical parameters, $\lambda$ and $\mu$ the Lamé parameters. Apart from the dielectric elasticity, the viscoelastic effect in the dielectric material is accounted for by means of the first Piola-Kirchhoff stress $\mathbf{P}^{\mathrm{vis}}$, see [16],

$$
\mathbf{P}^{\mathrm{vis}}=\frac{1}{2} J \eta\left(\mathbf{F}^{-T} \cdot \dot{\mathbf{F}}^{T} \cdot \mathbf{F}^{-T}+\dot{\mathbf{F}} \cdot \mathbf{C}^{-1}\right)
$$

in which $\eta$ is the damping parameter.

The strain energy function for the beam corresponding to the continuum model in Eq. (21) can be derived

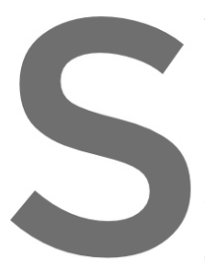

by integrating $\Omega\left(\mathbb{C}, \mathbb{E}^{e}\right)$ over the beam cros sectio
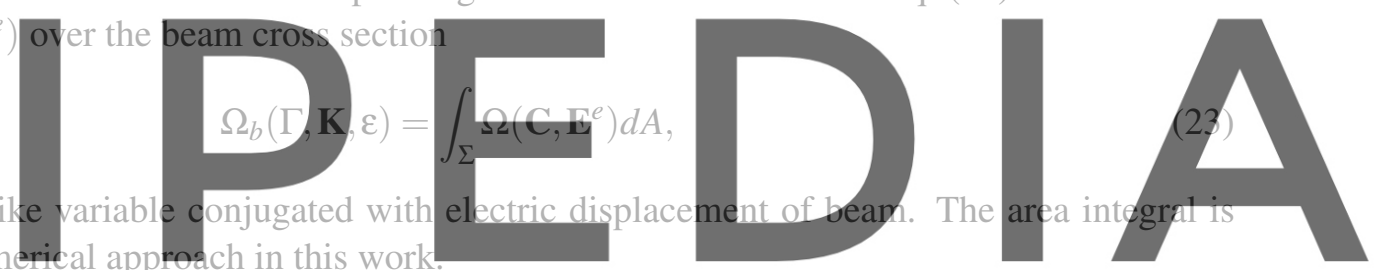

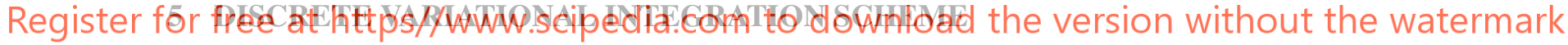

In this work, the electromechanically coupled beam dynamics is approximated using a constrained dis-

crete variational scheme. The Lagrange-d'Alembert principie for the constrained system reads

$$
\delta \int_{0}^{T}\left[L(\mathbf{q}, \dot{\mathbf{q}})-\mathbf{g}^{T}(\mathbf{q}) \cdot \lambda\right] d t+\int_{0}^{T} \mathbf{f}^{\mathrm{ext}}(t) \cdot \delta \mathbf{q} d t=0,
$$

with $\mathbf{q}$ the configuration, $L(\mathbf{q}, \dot{\mathbf{q}})$ the Lagrangian, $\mathbf{g}$ the holonomic constraints, $\lambda$ the Lagrangian multiplier and $\mathbf{f}^{\mathrm{ext}}(t)$ the external load. By treating the electric potential $\phi_{o}$ and the incremental variables $(\alpha, \beta)$ as the electric degrees of freedom $\phi=\left[\begin{array}{lll}\phi_{o} & \alpha & \beta\end{array}\right]$, the configuration of the beam model is extended to

$$
\mathbf{q}=\left[\begin{array}{lllll}
\varphi & \mathbf{d}_{1} & \mathbf{d}_{2} & \mathbf{d}_{3} & \phi
\end{array}\right]^{T} .
$$

According to the kinematic assumptions in geometrically exact beam, the directors have to fulfill the orthonormality. The continuous Lagrangian is composed by the kinetic energy $T(\dot{\mathbf{q}})$ and the internal potential energy $V(\mathbf{q})$ with

$$
L(\mathbf{q}, \dot{\mathbf{q}})=T(\dot{\mathbf{q}})-V(\mathbf{q}) .
$$


Since the electric variables do not contribute to the kinetic energy, the kinetic energy for the geometrically exact beam is computed as

$$
T=\int_{l}\left(\frac{1}{2} A_{\rho}|\dot{\varphi}|^{2}+\frac{1}{2} \sum_{i=1}^{2} M_{\rho}^{i}\left|\dot{\mathbf{d}}_{i}\right|^{2}\right) d s
$$

where $A_{\rho}$ is the mass density per reference arc-length and $M_{\rho}^{i}$ are the principle mass moments of inertia of cross section.

For the coupled hyperelastic material in DEA, the internal potential energy is computed by an integration of the beam strain energy density $\Omega_{b}$ in Eq. (23) over the line of centroids

$$
V(\mathbf{q})=\int_{l} \Omega_{b}(s) d s .
$$

The external load $\mathrm{f}^{\mathrm{xt}}$ contains all non-conservative loading, such as the gravity and the viscoelastic effect in this work. Based on the Kelvin-Voigt model in Eq. (22), the non-conservative work contributed by the viscoelastic effect is given by

$$
W^{\mathrm{vis}}=\int_{\Omega} \mathbf{P}^{\mathrm{vis}}: \mathbf{F} d V
$$

In this case, the external load corresponding to the viscoelastic effect can be formulated as
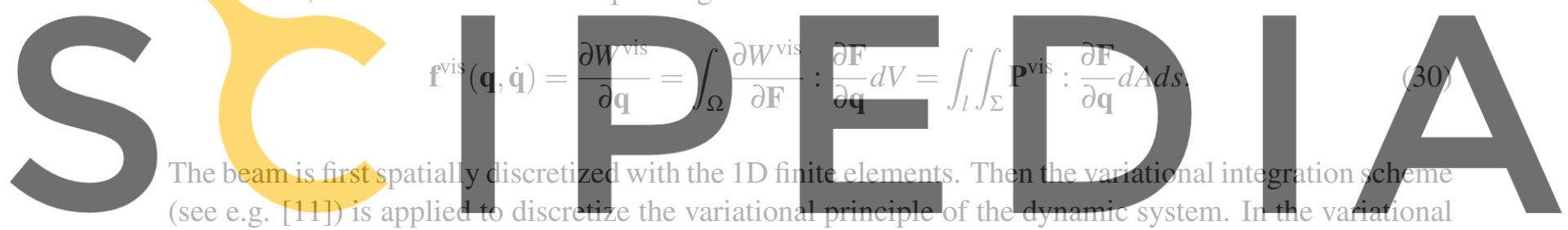

time integration scheme, the action integral within the time interval $\left(t_{n}, t_{n+1}\right)$ is approximated with the Register foirsfireel adrandjos/t/dwww.scipedia.com to download the version without the watermark

$$
\int_{t_{n}}^{t_{n+1}} L(\mathbf{q}, \dot{\mathbf{q}}) d t \approx L_{d}\left(\mathbf{q}_{n}, \mathbf{q}_{n+1}\right)=\Delta t L\left(\frac{\mathrm{q}_{n+1}+\mathrm{q}_{n}}{2}, \frac{\mathrm{q}_{n+1}-\mathrm{q}_{n}}{\Delta t}\right),
$$

where the discrete Lagrangian $L_{d}$ is computed by applying the finite difference approximation to the velocity $\dot{\mathbf{q}}$ and the midpoint rule to the configuration $\mathbf{q}$, i. e.

$$
\dot{\mathbf{q}} \approx \frac{\mathbf{q}_{n+1}-\mathbf{q}_{n}}{\Delta t}, \quad \mathbf{q} \approx \frac{\mathbf{q}_{n+1}+\mathbf{q}_{n}}{2} .
$$

To eliminate the constraint forces $\lambda$ from the system, the null space method, see e.g. [5], is applied. The discrete Euler-Lagrangian equations can be obtained by taking the variation of the discrete action and requiring stationarity. The nonlinear equation system is solved by use of the Newton-Rapson algorithm. In this work, the residual vector and the tangent matrix are derived by using the automatic differentiation tool CasADi [1]. 


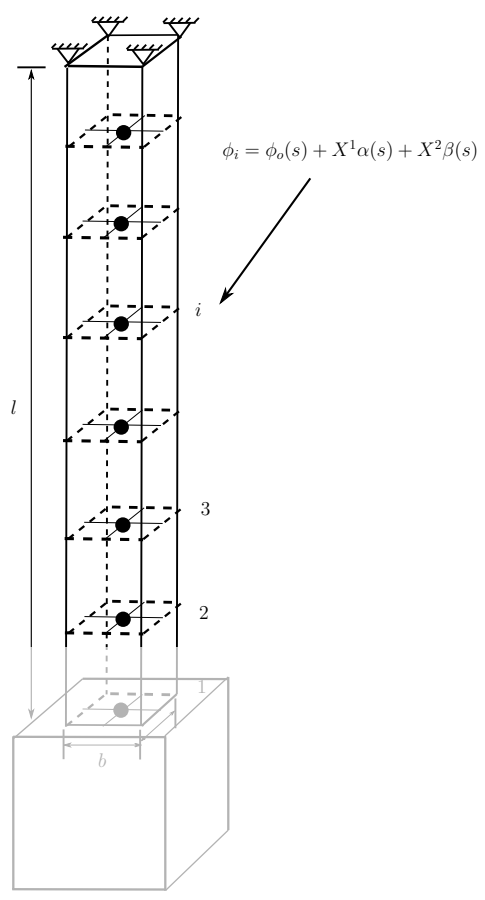

Figure 3: Boundary conditions on the beam.
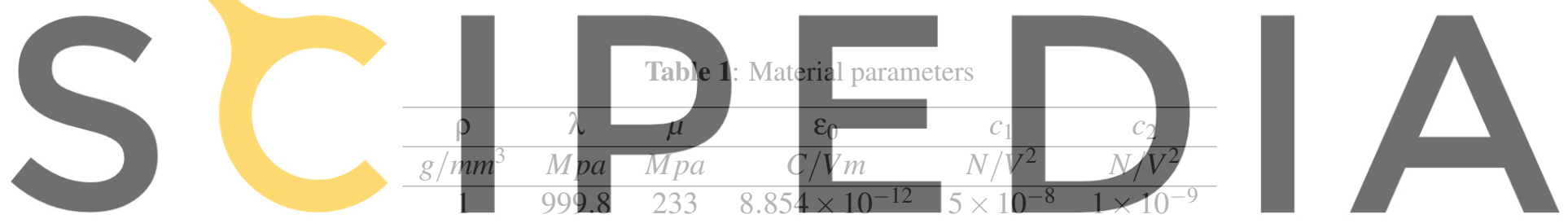

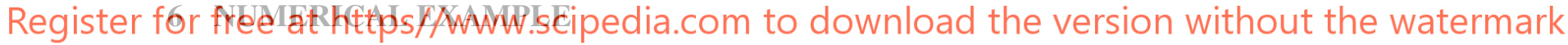

As a numerical example, a beam fixed at one end is considered to simulate a soft robotic arm holding a package as shown in Fig. 3. The package is represented by an additional point mass concentrated at the beam's free end. The beam has the length $l=0.1 \mathrm{~mm}$ and a square cross section with width $b=0.005 \mathrm{~mm}$. The material parameters of the dielectric elastomer applied in this work are shown in Table 1.

To conduct a specific task, different deformation modes have to be generated in the robotic arm. Thus, different electric boundary conditions $\phi_{i}$ will be imposed on nodes $i$ such that the desired deformations in the beam can be generated. For instance, the uniaxial contraction can be generated by applying a constant electric potential on the cross section, i.e. $\alpha, \beta=0$.

In this example, the motion of the beam discretized with 21 nodes is computed with the time step of $1 \times 10^{-3} \mathrm{~ms}$. As shown in Fig. 4(a) and 4(b), the package is fist lifted by the uniaxial contraction of the beam, where the constant electric potentials $\phi_{1}=8 \times 10^{4} \mathrm{~V}$ and $\phi_{21}=0 \mathrm{~V}$ are applied to the beam's fixed and free end, respectively, until $t=0.2 \mathrm{~ms}$. Then, from $t=0.201 \mathrm{~ms}$ to the end time, the bending of the beam is generated by changing the electric boundary conditions to $\phi_{i}=4 \times 10^{3}+3 \times 10^{4} X^{1} V(i=2,4, \ldots)$ and $\phi_{i}=0 V(i=1,3, \ldots)$, which leads to the moving of the package from ground to a desk, as shown in 
Fig. 4(c) and 4(d). In the simulation, the damping effect is imposed by setting $\eta=0.6$ in the viscoelastic model such that the beam is gradually approaching a steady state.

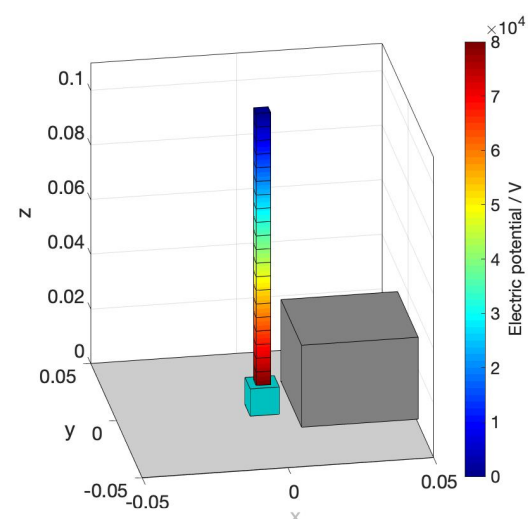

(a) $t=0 m s$
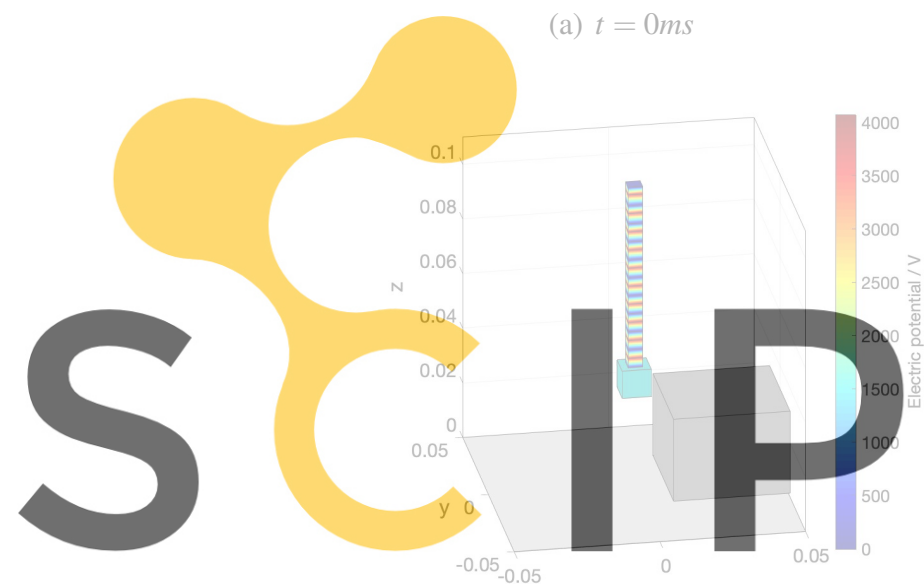

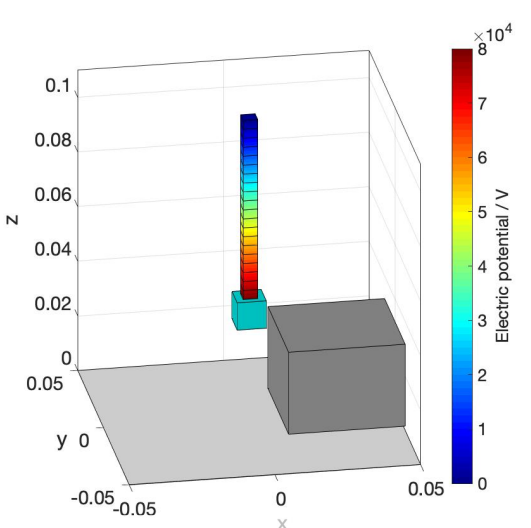

(b) $t=0.2 \mathrm{~ms}$

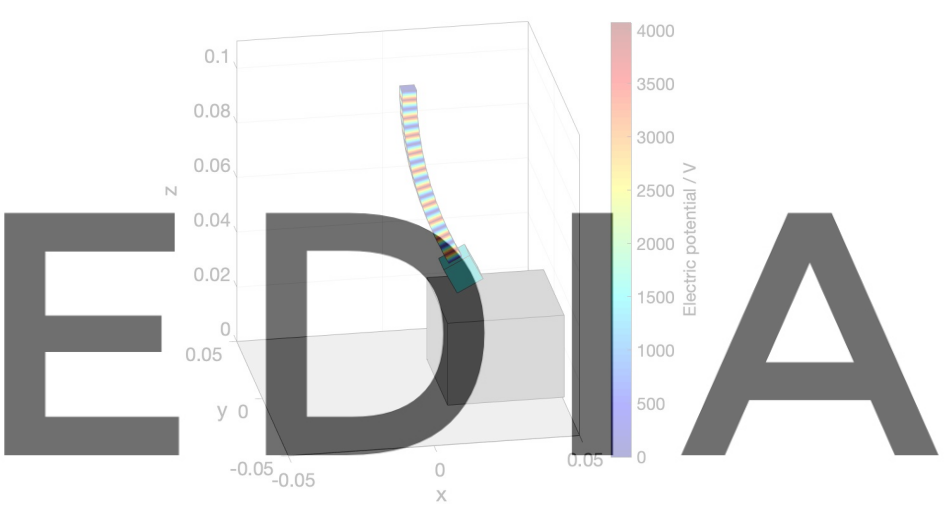

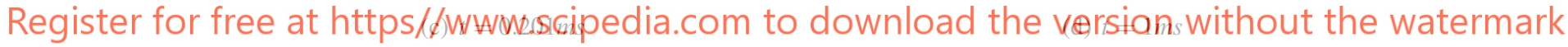

Figure 4: A soft robotic arm moving a package from the ground to a desk.

\section{CONCLUSIONS}

In this work, an electromechanically coupled beam model is developed. The proposed formulation of electric potential allows for different types of the dielectrically induced deformations in the beam, such as contraction and bending. The damping effect in the motion of the beam is accounted for by introducing a viscoelastic effect. The effectiveness of the developed beam model for DEA is demonstrated in a numerical example, where a soft robotic arm holding a package is presented.

\section{ACKNOWLEDGMENTS}

The authors acknowledge the support of Deutsche Forschungsgemeinschaft (DFG) for the project LE 1841-/5-11. 


\section{References}

[1] Joel A E Andersson, Joris Gillis, Greg Horn, James B Rawlings, and Moritz Diehl. CasADi - A software framework for nonlinear optimization and optimal control. Mathematical Programming Computation, 11(1):1-36, 2019.

[2] S.S. Antman. Nonlinear Problems of Elasticity. Springer, 2005.

[3] F Auricchio, P Carotenuto, and A Reali. On the geometrically exact beam model: a consistent, effective and simple derivation from three-dimensional finite-elasticity. International Journal of Solids and Structures, 45(17):4766-4781, 2008.

[4] Yoseph Bar-Cohen. Electroactive polymers as artificial muscles: capabilities, potentials and challenges. In Robotics 2000, pages 188-196, 2000.

[5] Peter Betsch and Sigrid Leyendecker. The discrete null space method for the energy consistent integration of constrained mechanical systems. part ii: Multibody dynamics. International journal for numerical methods in engineering, 67(4):499-552, 2006.

[6] Eugene Cosserat and François Cosserat. Théorie des corps déformables. A. Hermann et fils, 1909.

[7] A Dorfmann and RW Ogden. Nonlinear electroelasticity. Acta Mechanica, 174(3-4):167-183, 2005.

[8] Mihai Duduta, Ehsan Hajiesmaili, Huichan Zhao, Robert J Wood, and David R Clarke. Realizing the potential of dielectric elastomer artificial muscles. Proceedings of the National Academy of
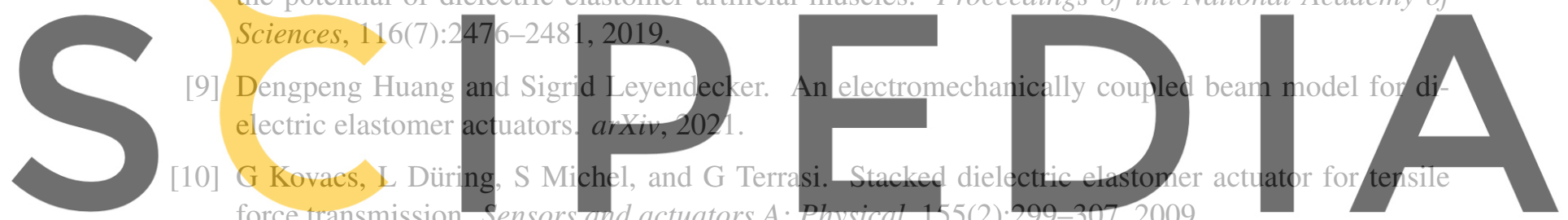
force transmission. Sensors and actuators $A$ :

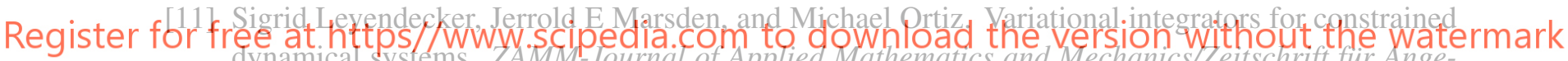 dynamical systems. ZAMM-Journat of Applied Mathematics and Mechanics/Leitschrift fur Ange- wandte Mathematik und Mechanik: Applied Mathematics and Mechanics, 88(9):677-708, 2008.}

[12] C Löwe, X Zhang, and G Kovacs. Dielectric elastomers in actuator technology. Advanced Engineering Materials, 7(5):361-367, 2005.

[13] Y. H. Pao. Electromagnetic forces in deformable continua. In Mechanics today. (A78-35706 14-70) New York, volume 4, pages 209-305, January 1978.

[14] Ronald E Pelrine, Roy D Kornbluh, and Jose P Joseph. Electrostriction of polymer dielectrics with compliant electrodes as a means of actuation. Sensors and Actuators A: Physical, 64(1):77-85, 1998.

[15] T Schlögl and S Leyendecker. Dynamic simulation of dielectric elastomer actuated multibody systems. In Smart Materials, Adaptive Structures and Intelligent Systems, volume 50480, page V001T02A003. American Society of Mechanical Engineers, 2016.

[16] T Schlögl and S Leyendecker. A polarisation based approach to model the strain dependent permittivity of dielectric elastomers. Sensors and Actuators A: Physical, 267:156-163, 2017. 
[17] Tristan Schlögl and Sigrid Leyendecker. Electrostatic-viscoelastic finite element model of dielectric actuators. Computer Methods in Applied Mechanics and Engineering, 299:421-439, 2016.

[18] JC Simo. Finite strain beam theory. Comput. Methods Appl. Mech. Eng, 49:55-70, 1985.

[19] Zhigang Suo. Theory of dielectric elastomers. Acta Mechanica Solida Sinica, 23(6):549-578, 2010.

[20] Zhigang Suo, Xuanhe Zhao, and William H Greene. A nonlinear field theory of deformable dielectrics. Journal of the Mechanics and Physics of Solids, 56(2):467-486, 2008.

[21] DK Vu, P Steinmann, and G Possart. Numerical modelling of non-linear electroelasticity. International Journal for Numerical Methods in Engineering, 70(6):685-704, 2007.

[22] Michael Wissler and Edoardo Mazza. Electromechanical coupling in dielectric elastomer actuators. Sensors and Actuators A: Physical, 138(2):384-393, 2007.

[23] Xuanhe Zhao, Wei Hong, and Zhigang Suo. Electromechanical hysteresis and coexistent states in dielectric elastomers. Physical review B, 76(13):134113, 2007. 